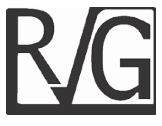

\title{
De la innovación a la ecoinnovación. Gestión de servicios en empresas hoteleras
}

\author{
Velázquez-Castro, Judith Alejandra* \\ Vargas-Martínez, Elva Esther"
}

\section{Resumen}

Organismos internacionales como la Agencia Finlandesa de Financiación de Tecnología e innovación o la Universidad de las Naciones Unidas - Maastricht Instituto Económico de Investigaciones Sociales de Innovación y Tecnología (UNU-MERIT), han propuesto modelos de innovación para empresas de servicios; no obstante, son escasos los estudios que hacen referencia a procesos de innovación ambiental. En México, el sector turístico está integrado en su mayoría por empresas de hospedaje con condiciones estructurales y de gestión para impulsar nuevos esquemas de negocio, basados en la ecoinnovación. Así, se analizan modelos de innovación en servicios formulados por organismos públicos y privados, que pueden ser aplicados en hoteles. La finalidad es proponer un modelo de ecoinnovación que sobre la base de la vigilancia tecnológica hasta la evaluación del desempeño ambiental, logre un proceso de innovación que contribuya con la competitividad empresarial a la vez que con el cuidado ecológico. Se realizó un análisis de literatura para identificar la naturaleza del sector servicio, así como distintos modelos de innovación. En última instancia se conjuntan elementos de gestión ambiental y de gestión tecnológica, para llegar a la propuesta del modelo de ecoinnovación. Como conclusión, se verifica la solidez conceptual y metodológica del modelo, no obstante, puede ser mejorado y validar su utilidad práctica empresarial a partir de su aplicación, para diseñar un plan de fortalecimiento a la gestión ambiental y de innovación.

Palabras clave: Innovación; servicios; ecoinnovación; gestión de empresas hoteleras.

\section{From innovation to ecoinnovation. Service management in hotel companies.}

\section{Abstract}

International organisms such as the Finnish Funding Agency for Technology and Innovation or the United Nations University - Maastricht Economic and Social Research Institute on Innovation

\section{Recibido: 02.02.15. Aceptado: 10.05.15}

* Doctora en Estudios Turísticos, profesora de la Universidad Autónoma del Estado de Hidalgo, email: judithalejandra666@gmail.com.

** Doctora en Ciencias Ambientales, profesora investigadora y coordinadora del CIETUR, Facultad de Turismo y Gastronomía, Universidad Autónoma del Estado de México. 
and Technology (UNU-MERIT) have proposed innovation models for service companies; nevertheless, the studies making reference to environmental innovation processes are scarce. In México, the touristic sector is composed in its majority by hosting companies with structural and management conditions to impulse new business schemes based on the ecoinnovation. Thus the innovation models on services formulated by public and private organisms that could be applied on hotels are analyzed. The aim is to propose an ecoinnovation model on the base of technology surveillance up to the environmental performance evaluation that contributes to the business competitiveness and at the same time to the ecological care. A literature analysis was made in order to identify the nature of the service sector as well as distinct innovation models. Ultimately, environmental management elements and technological management come together to achieve the proposal of the ecoinnovation model. As conclusion, the conceptual and methodological solidity of the model is verified, nonetheless, it could be improved and validate its practical business utility from its application with the intention of designing a strengthening plan for the environmental and innovation management.

Key words: Innovation; service; eco-innovation; hotel management companies.

\section{Introducción}

Por años, el turismo como actividad económica empresarial, ha demostrado su capacidad y dinamismo por tener un rápido crecimiento en el mundo, no obstante, hoy en día se encuentra en un momento crucial de desarrollo, donde los aspectos de rentabilidad económica, inclusión social y responsabilidad ambiental están siendo cada vez más decisivos para el logro de su competitividad (World Commision on Environment and Development, 1987).

Desde la última década se ha venido demandando a las empresas turísticas el cumplimiento con la diversidad biológica y los sistemas que dan soporte a la vida (Organización Mundial del Turismo -OMT, 1998:102); además, su escenario futuro está inmerso en un nuevo panorama competitivo, donde la tecnología y la innovación, se articulan para dar pie a una nueva dinámica de gestión empresarial que permita a las organizaciones afrontar los cambios y desafíos del entorno que las rodea.

Autores como Zahra y George (2002), la Organización para la Cooperación y el Desarrollo Económicos (OCDE, 2012) o Aguilar et al, (2013), señalan que las empresas del sector servicios dan menos importancia a factores relacionados con la adquisición, aplicación de tecnologías, o empleo de conocimiento e información. Temas como la ecoeficiencia o la ecoinnovación no han logrado ser apropiados por la hotelería, reforzando su falta de compromiso por el cuidado de la naturaleza (Días, 2008; Hernández y García, 2011; Velázquez y Vargas, 2014).

En contraparte, otros trabajos aseguran que en el sector turístico se desarrollan e implementan diversas innovaciones, pero a diferencia de otras industrias, en el turismo son más frecuentes y evidentes las innovaciones tecnológicas blandas ${ }^{1}$ o gestionales (Fundación COTEC

1 Las tecnologías blandas, llamadas también gestionales, se ocupan de la transformación de elementos simbólicos en bienes y servicios; su producto, que no es un elemento tangible, permite mejorar el funcionamiento de las instituciones y organizaciones para el logro de sus objetivos (Tecuanhuey, 2007:1).

2 Las tecnologías duras son las que tienen como propósito la transformación de elementos materiales, con el fin de producir bienes y servicios. Entre ellas pueden distinguirse dos grandes grupos: las que producen objetos sobre la base de acciones físicas sobre la materia y las que basan su acción en procesos químicos y/o biológicos (Salazar, Romero y Carranza, 2010:11). 
De la innovación a la ecoinnovación. Gestión de servicios en empresas hoteleras Velázquez-Castro, Judith Alejandra y Vargas-Martínez, Elva Esther

para la Innovación Tecnológica, 2010). Del mismo modo, en el turismo también se implementan innovaciones tecnológicas duras $^{2}$ transferidas desde otros sectores.

Existen variados modelos que permiten gestionar la innovación en las empresas de servicios; sin embargo, para llegar a una propuesta de modelo de ecoinnovación para los hoteles, se deben precisar las particularidades que determinan la propensión a innovar en la esfera ambiental. Así, el presente artículo parte del interés de acercarse a estos planteamientos, realizando un análisis de los modelos de innovación en servicios formulados tanto por organismos públicos como privados, y que pueden ser aplicados en los hoteles; se identifican las propiedades para intervenir en el proceso de innovación, y se estudian las relaciones que se establecen entre ellas con la gestión ambiental para lograr un cambio o mejora en el producto, servicio o proceso organizacional.

Se pretende construir la propuesta de un modelo de ecoinnovación para empresas hoteleras, que sobre la base de la vigilancia tecnológica hasta la evaluación del desempeño ambiental, logre un proceso de innovación que contribuya tanto a la competitividad empresarial como al cuidado ecológico; todo ello adoptando una concepción sistémica de la organización.

La metodología para la
operacionalización del modelo de
ecoinnovación consistió en primera
instancia en la revisión de la literatura, en donde se analizan las características y naturaleza del sector servicios; asimismo se identifican diversos modelos de innovación en servicios, incluido el Modelo de Gestión Tecnológica e innovación; en segundo término se reconocen las particularidades de las empresas hoteleras; en una tercera etapa se conjuntan los elementos de gestión ambiental y de gestión de la ecoinnovación. Por lo que, el modelo de ecoinnovación propuesto consiste en la conexión esquemática de 4 funciones que sobre la base de la gestión ambiental y la innovación, consisten en: 1) Vigilar, 2) Planear y habilitar, 3) Implantar y 4) Verificar y evaluar. Asimismo, el modelo refleja la lógica de una empresa para alcanzar sus retribuciones económicas, cubriendo las áreas principales: cliente, oferta, infraestructura, viabilidad económica y desempeño ambiental.

\section{Innovación en servicios}

En contraste con la producción de bienes materiales, en los servicios no se aplica el conocimiento o experiencia de las técnicas de fabricación directamente; es preciso idear técnicas de gestión especializadas y distintas para su adecuada operación. Asimismo, los servicios necesitan la presencia o interacción del cliente, esto, debido a las diferentes demandas que deben ser atendidas con acciones y que requieren del cliente en el proceso de prestación o consumo

De acuerdo con Camisón et al, (2007) y Betancourt y Mayo (2010), las principales características de los servicios, que los distinguen de los bienes materiales y que tienen implicaciones directas en la definición y análisis de la innovación, son: 1) la relación estrecha entre producción y consumo; 2) intangibilidad y contenido intensivo en información de los productos y procesos en servicios; 3) papel fundamental de los recursos humanos como factor básico de competitividad; e 4) importancia de los factores organizativos para el performance de la empresa.

Así, la intangibilidad es la principal característica que distingue 
a los servicios de los bienes, ya que se ofrecen beneficios o satisfacciones para su venta. Otra característica, es la capacidad productiva del proveedor, en la que tiene contacto directo con el cliente y en la que se refleja la entrega simultánea y el consumo de los servicios -aquí, el consumidor puede afectar o determinar el rendimiento y la calidad del servicio prestado-. También se considera la fuerte participación de recursos humanos en la operación del servicio que al ser entregado por diferentes personas, puede llegar a existir variabilidad; esta característica ofrece la oportunidad de proporcionar un grado de flexibilidad y personalización del servicio, por lo que también puede ser un beneficio y punto de diferenciación.

Por último, la actuación de la empresa considera el rendimiento, competencia, desempeño y realización de la organización (Arribas, 2010). Esta característica considera el trabajo para la prestación del servicio y la innovación del mismo; ya que la innovación en sí misma promueve calidad y competitividad.

Dada la naturaleza de los servicios, el Ministerio de Industria y Comercio de Noruega (2006:10-11) propone la siguiente clasificación, útil en la comprensión del por qué y cómo innovan las empresas, distinguiendo esencialmente cuatro grupos de empresas (cuadro 1):

\section{Cuadro 1}

\section{Tipología de las empresas prestadoras de servicios}

\begin{tabular}{ll}
\hline \multicolumn{1}{c}{ Grupo } & \multicolumn{1}{c}{ Características } \\
\hline $\begin{array}{l}\text { Solucionadores } \\
\text { de problemas }\end{array}$ & $\begin{array}{l}\text { Empresas que brindan soluciones a dificultades específicas de otras empresas; } \\
\text { ello implica escasa o nula posibilidad de estandarización. }\end{array}$ \\
$\begin{array}{l}\text { Servicios de } \\
\text { asistencia }\end{array}$ & $\begin{array}{l}\text { Se especializan en la oferta de servicios de consumo, tales como seguridad, } \\
\text { limpieza, entre otros. }\end{array}$ \\
$\begin{array}{l}\text { Servicios de } \\
\text { distribución }\end{array}$ & $\begin{array}{l}\text { Se encargan del suministro y transporte de bienes y materias primas, pasajeros } \\
\text { e información. En este grupo existen proveedores manuales y digitales, que } \\
\text { buscan facilitar la interacción entre los clientes. }\end{array}$ \\
$\begin{array}{l}\text { Servicios de } \\
\text { ocio }\end{array}$ & $\begin{array}{l}\text { Empresas dedicadas a estimular las emociones de los consumidores, por } \\
\text { medio de la oferta de actividades como deportes, arte, entretenimiento, } \\
\text { alimentos y bebidas, hospedaje, cultura, entre otros. }\end{array}$ \\
\hline
\end{tabular}

Fuente: Ministerio de Industria y Comercio de Noruega (2006:10-11).

Al considerar que la innovación en servicios es impulsada por diversos propósitos, y que cubre básicamente todas las medidas que contribuyan a incrementar la rentabilidad de la empresa y a un mayor valor añadido para el cliente, a través de la renovación y mejora, la tipología anterior lista los distintos tipos de servicios -desde la limpieza, las telecomunicaciones, hasta el ocio-, considerando la diversidad de los mismos y la heterogeneidad de las innovaciones en este sector. 
De la innovación a la ecoinnovación. Gestión de servicios en empresas hoteleras Velázquez-Castro, Judith Alejandra y Vargas-Martínez, Elva Esther

\subsection{Proceso de innovación en servicios}

De acuerdo con Hinojosa (2006), el proceso de innovación se compone de:

1) Diagnóstico. De los factores que intervienen en el funcionamiento de la organización; con el propósito de elaborar un pronóstico a corto o medio plazo a partir de la tendencia existente o la previsión de futuros cambios. Es necesario conocer cuáles son las necesidades de servicio, qué productos son demandados a la empresa, y se detectan debilidades que se transforman en fortalezas.

2) Identificación de oportunidades. Para el desarrollo de procedimientos, productos o servicios de acuerdo con las necesidades detectadas en la fase anterior.

3) Capacidad técnica y administrativa de la empresa para satisfacer esa necesidad. Proceso de transformación del conocimiento y tecnología adquirida por la empresa. Los estudios de mercado y de los consumidores deberán determinar si la innovación es compatible o no con el estado actual de las cosas.

Tomando en consideración lo antepuesto, se infiere que la innovación surge a partir de la creatividad y cultura generada por la propia empresa, en donde los recursos humanos son la base para tal acción. Cabe mencionar que un buen clima organizacional propicia el espíritu innovador en los empleados; por lo que, es necesario un ambiente que permita a la gente imaginar, crear y producir.

Es así que Schilling y Werr (2009) proponen un proceso que toma como base la creación y gestión del conocimiento, ya que éste es el fundamento para crear algo nuevo. Tal y como lo muestra el diagrama 1, este proceso está compuesto por ocho fases que giran en torno a la gestión del conocimiento (Schilling y Werr, 2009):

\section{Diagrama 1 Proceso de innovación}

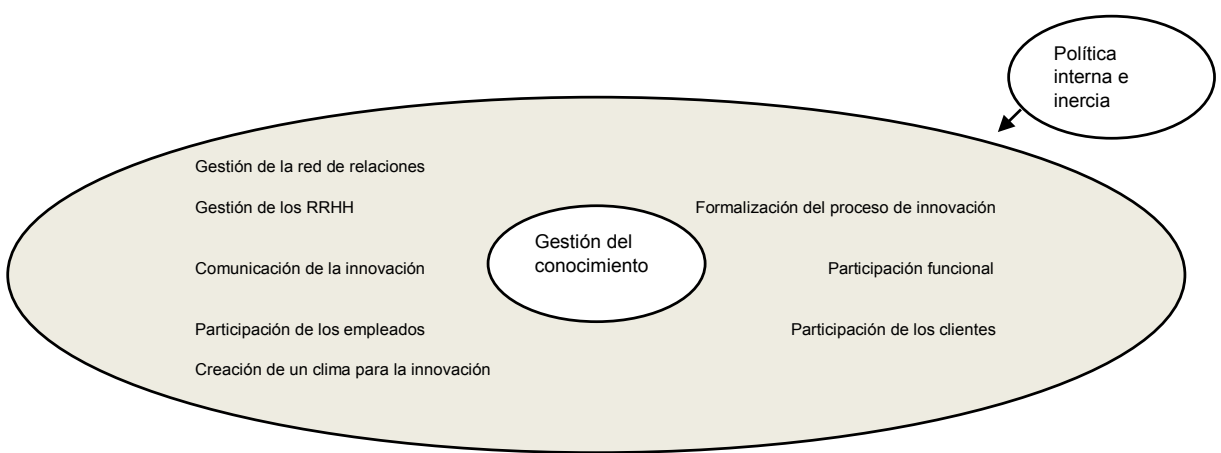

Fuente: Schilling y Werr, 2009. 
A diferencia del proceso propuesto por Hinojosa (2006), Schilling y Werr (2009) enfatizan en la gestión del conocimiento, al ser una de las principales estrategias para la administración de personal, que implica gestionar los procesos de creación, desarrollo, difusión y explotación del conocimiento para dominar la capacidad organizativa e introducir procesos creativos; además, identifica el intercambio de ideas y conocimientos valiosos ya existentes en la organización. Así, una empresa innovadora considera que la gestión y creación de conocimiento es importante para dar lugar a nuevas ideas de servicios o prácticas organizacionales.

\section{Modelos de gestión de la innovación en servicios}

En un sentido amplio, la gestión de la innovación consiste en introducir una novedad en la organización y, como tal, representa una forma particular de cambio, bajo ese argumento, los modelos de innovación pueden utilizarse como apoyo para la gestión en servicios, interacción con el cliente, control de calidad o mejoramiento de las actividades internas de la empresa.

A nivel mundial, seis de los países con mayor nivel de innovación ${ }^{3}$ se encuentran en la Unión Europea (UE), por lo que los modelos de innovación más relevantes para el sector servicios que se presentan en este apartado, son de países de esa región (EKOS Negocios, 2011:42). A continuación se explican los modelos propuestos:

\section{a) Modelo dimensional de la innovación en servicios}

Llamado así porque simplifica las diversas variables involucradas en el proceso de innovación en servicios. Como resultado fundamental, se tiene el entendimiento de estos parámetros, obteniéndose combinaciones adecuadas y necesarias para estudiar cada uno de sus procesos. De este modo, se consigue analizar con mayor facilidad todo el sistema de innovación de una empresa de servicios, reducir el tiempo y el número de actividades, y conocer mejor su comportamiento.

En el contexto anterior, Hertog y Bilderbeek (1998) presentan un modelo de cuatro dimensiones para la innovación en servicios (diagrama 2); cada dimensión aborda un área distinta de la empresa. Iniciando con la concepción de un nuevo servicio (dimensión 1), aquí las innovaciones pueden ser visibles, pero habitualmente consisten en una nueva forma de organizar una actividad para solucionar un problema. Es esencial que su aplicación sea novedosa en un mercado definido.

La dimensión 2, tiene que ver con una nueva interfaz con el cliente, en donde los productos se ofrecen y comercializan orientados de forma específica a grupos de consumidores, lo cual acentúa la importancia de la comunicación entre los proveedores y los clientes. La dimensión 3, consiste en un nuevo sistema de provisión (distribución y entrega) del servicio; y se centra en la relación entre el proveedor y sus clientes, así como en los cambios organizativos -internos- que permiten

3 Los países con mayor nivel de innovación son: 1.Suiza, 2.Suecia, 3.Singapur, 4. Hong Kong, 5. Finlandia, 6. Dinamarca, 7. Estados Unidos, 8. Canadá, 9. Holanda y 10. Reino Unido (EKOS Negocios, 2011:42). 
De la innovación a la ecoinnovación. Gestión de servicios en empresas hoteleras Velázquez-Castro, Judith Alejandra y Vargas-Martínez, Elva Esther

mejorar la manera en la que el personal desarrolla sus actividades para ofrecer los servicios de una manera más adecuada.

Por último, la dimensión 4 contempla las

opciones tecnológicas para el

servicio, tales como las tecnologías de la información y la comunicación (TIC); en el cual el conocimiento juega un importante papel para cada proceso.

\section{Diagrama 2 \\ Modelo dimensional de la innovación en servicios}

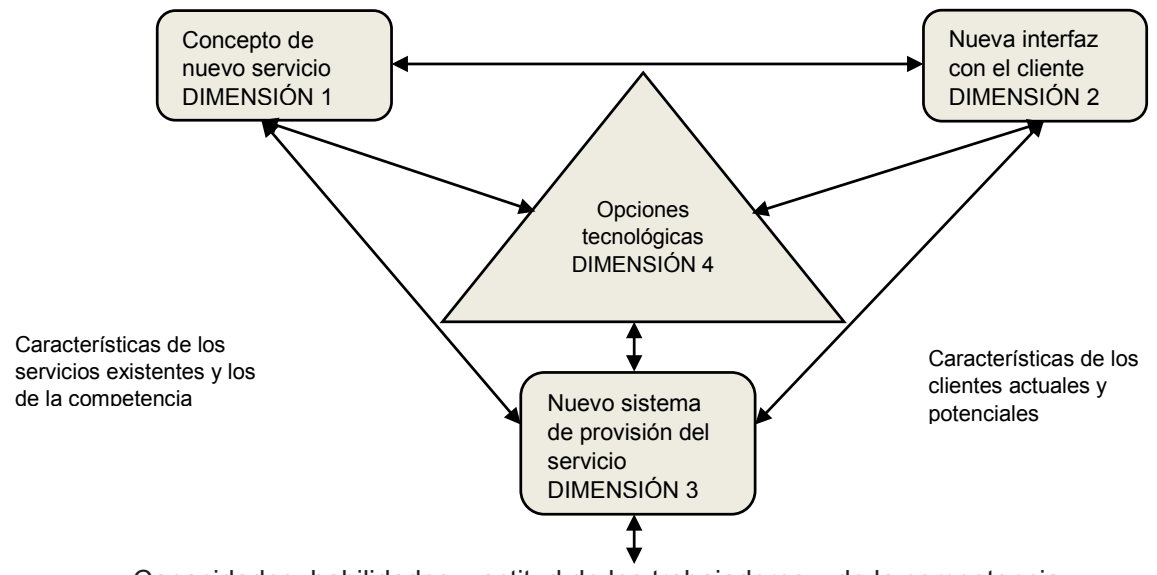

Capacidades, habilidades y actitud de los trabajadores y de la competencia.

Fuente: Den y Bilderbeek (1998).

\section{b) Modelo Finlandés}

El modelo gestión de la innovación de Finlandia, surge para ayudar a las empresas de ese país en el desarrollo de las principales áreas de tecnología. EI gobierno finlandés detectó que la mejora de la tecnología tiene un papel clave en el resurgimiento económico, y desde la década de 1990 a la fecha, se ha duplicado el apoyo para proyectos de investigación realizados por universidades, institutos de investigación y empresas, cuya finalidad son crear nuevas competencias y soluciones para los problemas de su organización (Finnish Funding Agency for Technology and Innovation, TEKES: 2014).
El modelo contempla dos etapas: inside-out, que refleja el concepto tradicional de la cadena de valor; y outside-in, en el que se hacen cambios en la perspectiva tradicional y se procura recoger las demandas de los consumidores, de forma tal, que la organización pueda desarrollar cambios en su modelo de negocio.

En la primera etapa, se describen los procesos que agregan valor a la empresa y la manera en que la empresa organiza sus recursos a favor de la innovación. Enseguida, se mencionan las características básicas del performance empresarial y del servicio al cliente. Como tercer paso, se habla de la distribución del servicio, 
es decir, la forma en la que se enlaza éste con el cliente; también se considera la importancia que tiene la marca para generar valor agregado para el cliente, y por último, la etapa financiera que hace referencia al modelo de negocio de la empresa, así como la importancia de su cadena de valor.

\section{c) Modelo House of Innovation}

Este modelo está basado en una visión holística y sistemática del denominado ecosistema de la innovación; es decir, en el conjunto de diferentes elementos que se interrelacionan entre sí para generar una ventaja competitiva. El modelo ha sido construido en forma piramidal, teniendo como base a los facilitadores de la innovación -recursos humanos, conocimiento, proyectos $y$ programas-, y en la cima, la estrategia de innovación (ATKearney, 2007). En cuanto a la gestión de los facilitadores de la innovación, ésta se produce utilizando diversas técnicas, tales como la gestión del conocimiento, de las capacidades en tecnologías específicas o de la experiencia en una nueva habilitación de desarrollo del mercado; asegurando la gestión de los proyectos $y$ el control de los recursos con la aplicación de herramientas tecnológicas y compartiendo conocimiento.

En el segundo peldaño, se ubica la gestión del ciclo de vida de la innovación; para ello se requiere gestionar las ideas, desarrollar productos y procesos, y aplicar la mejora continua. Estas tareas pueden llevarse a cabo por medio de la sistematización del proceso de generación de ideas y de innovación para crear procesos continuos de mejora, acelerando su introducción al mercado. Como tercer escalón, se encuentra la organización de la innovación y la cultura; lo que permite involucrar a la alta gerencia de la empresa con el resto de la organización; promoviendo una cultura a partir de la identificación de fallas y errores, toma de decisiones y aprovechamiento de recursos internos y externos.

En la cima se localiza la estrategia de innovación, que supone la creación de una visión clara de la innovación, que debe estar alineada a la estrategia del negocio; garantizando la comunicación en todas las escalas; analizando las tendencias del entorno; y midiendo los logros alcanzados con base en los objetivos estratégicos.

\section{d) Modelo Nacional de Gestión de Tecnología e Innovación}

El Modelo Nacional de Gestión de Tecnología e Innovación (MNGdTi), del Premio Nacional de Tecnología e Innovación (2012), tiene como propósito impulsar el desarrollo de las organizaciones mexicanas, de cualquier giro o tamaño, mediante la gestión tecnológica e innovación (GTi) explícita, sostenida y sistemática.

El modelo se fundamenta en la gestión de tecnología, ya que con base en ella, las empresas maximizan sus ventajas competitivas a través del uso sistemático de los medios tecnológicos y organizacionales necesarios para ello. La GTi, otorga congruencia sobre nuevos métodos para crear, transformar y entregar valor a los clientes. El modelo se integra por una serie de funciones y procesos de GTi, que incluye actividades para mejorar el trabajo administrativo que se realiza dentro de la organización, estas tareas pueden agruparse dado su similar naturaleza para el logro de 
De la innovación a la ecoinnovación. Gestión de servicios en empresas hoteleras Velázquez-Castro, Judith Alejandra y Vargas-Martínez, Elva Esther

un fin común. Cuando las actividades de GTi se realizan de forma secuencial, sistemática, tienen objetivos y metas claras, y muestran cómo las cosas cambian en el tiempo, constituyen la base de un proceso de GT.

El modelo consta de cinco funciones: 1) vigilar, consiste en la búsqueda de amenazas y oportunidades de desarrollo e innovación tecnológica que impacten sobre la empresa; 2) planear, el desarrollo de un marco estratégico tecnológico, que le permita a la organización seleccionar líneas de acción que deriven en ventajas competitivas; 3) habilitar, la obtención de tecnologías y recursos necesarios (dentro y fuera de la organización) para la ejecución de los proyectos a incluir; 4) proteger, es la salvaguarda y cuidado del patrimonio tecnológico de la organización; e 5) implantar, realización de los proyectos de innovación hasta el lanzamiento en el mercado de un servicio nuevo o mejorado, o la adopción de un proceso nuevo o sustancialmente mejorado. Incluye la explotación comercial de las innovaciones y las expresiones organizacionales que se desarrollan para ello.

Como se observa en los modelos anteriores, la naturaleza de las actividades o acciones de innovación emprendidas en el sector servicios, es distinta a la naturaleza de las realizadas por el sector industrial; lo anterior se debe a que el resultado final de los procesos de innovación en la industria, es un producto tangible, mientras que en los servicios, el producto final es inmaterial y elaborado y consumido in situ.

Por un lado, el modelo dimensional de la innovación en servicios y el de gestión de la innovación de Finlandia, suponen como fuentes de innovación las demandas de los consumidores, para crear nuevos mercados con nuevas reglas, determinando si la innovación es compatible o no con el estado actual de la empresa. En cuanto al modelo piramidal, éste considera que la gestión del conocimiento juega un importante papel para el desarrollo de cada proceso encaminado a la innovación. En este modelo, los recursos humanos son valorados, en el entendido de que los servicios son brindados directamente por los empleados de la empresa.

Por último, el modelo del PNT toma como base la gestión tecnológica, al ser considerada una herramienta que le permite a las empresas maximizar sus ventajas competitivas a través del uso sistemático de los medios tecnológicos y organizacionales. Sin embargo, la gestión del conocimiento toma un papel secundario (presentada como uno de los procesos de la función a habilitar).

Cabe resaltar que estos modelos, explican el proceso desde un enfoque holístico y sistémico; basándose en el aprovechamiento del conocimiento generado por la empresa o por otros agentes externos. No obstante, en ellos no se considera como parte integral ya sea de las funciones o procesos- el ámbito ecológico.

Por lo anterior, se determina que es indispensable un redireccionamiento de los modelos para garantizar y estimular las actividades de ecoinnovación en el sector servicios. Un modelo que le permita a la industria hotelera incluir a sus prácticas procesos innovadores para el cuidado ambiental, en virtud de la necesidad de impulsar nuevos procesos desde una perspectiva sustentable $y$ para el sostenimiento de la actividad turística en el largo plazo. 


\section{Determinantes para un modelo de ecoinnovación en empresas hoteleras}

Hellström (2007) ha referido el estrecho significado de la ecoinnovación como una simple reducción de los impactos ambientales a través de la minimización de residuos, no obstante la noción es mucho más amplia, ya que los resultados de una empresa ecoinnovadora pueden llegar a mejorar el uso del espacio y la preservación de la biodiversidad, hasta lograr incrementar la seguridad y la calidad de vida de los habitantes de los destinos turísticos.

A lo largo del tiempo, las organizaciones del sector turístico han optado por distintos modelos de gestión, generalmente basados en la mejora continua; estos modelos poseen la base para la gestión de la innovación, pero carecen de la articulación de los procesos de innovación para la solución de problemas ambientales. Así, en el servicio de alojamiento existen importantes razones para introducir ecoinnovaciones: hacer más eficiente el uso de instalaciones y equipos, reducir el consumos de energía y agua, disminuir los costos de operación, controlar la contaminación, producir servicios ecológicos, cumplir con la normativa ambiental, o mejorar la imagen de la empresa (Arundel y Kemp, 2009).

Así, para poner en contexto las prácticas y mecanismos de innovación de estas empresas, es preciso conocer los aspectos relacionados con su gestión: funciones de las áreas o departamentos - de soporte o de contacto directo con el cliente-, motivaciones que impulsan las actividades de innovación, barreras que pueden inhibir dichas prácticas, y demás recursos necesarios para su desarrollo.
Cabe resaltar, que la suposición de un modelo de innovación, describe la lógica de negocio detrás de la creación de valor para las empresas. Al final, un modelo viable y factible siempre debe entregar valor a los clientes, así como a los demás actores que participan en el sistema de creación de valor Actualmente, el sector turístico centra su atención en ofertar servicios mejorados, pero a menudo no incluye las variables ambiental y tecnológica dentro de su modelo de negocio. Por lo que, el modelo de ecoinnovación propuesto tiene como finalidad crear valor para los establecimientos de hospedaje, a partir de la inclusión de estas variables.

Es así, que la presente propuesta describe la manera en que las empresas crean valor a través del uso eficiente de los recursos naturales y tecnológicos. De esta forma, sobre la base de la Vigilancia Tecnológica, es necesario identificar las mejores tecnologías disponibles y buenas prácticas de gestión, para analizar el contexto espacial de las empresas, para conocer sí éstas pueden adaptarse a las características y necesidades típicas de los hoteles.

La ecoinnovación en los establecimientos de hospedaje, es concebida como la combinación entre los procesos de gestión ambiental y los procesos de innovación, para generar su adopción progresiva y facilitar el mejoramiento continuo de su desempeño. Por lo que, el modelo de ecoinnovación que se presenta, centra su atención en la mejora continua, a través del diseño o adquisición de innovaciones ambientales, adaptándose a los continuos cambios del mercado. Así entonces, se retoma el Modelo Nacional de Gestión de la Tecnología e Innovación del PNT; cuyo objetivo es evitar el estancamiento que se produce 
De la innovación a la ecoinnovación. Gestión de servicios en empresas hoteleras Velázquez-Castro, Judith Alejandra y Vargas-Martínez, Elva Esther

en las organizaciones, para facilitar la creatividad y ayudar al diseño de un nuevo programa de gestión de innovación; asimismo, se consideran algunas otras variables expuestas en los modelos de innovación en servicios analizados.

Cabe señalar, que a todos los departamentos de la organización les corresponde estar involucrados en tal proceso, en el entendido de que la ecoinnovación tendrá beneficios para todos. La propuesta se compone de una serie de funciones en donde se integran de forma complementaria la innovación tecnológica y la gestión ambiental en un modelo único, para impulsar la mejora continua de la empresa y estimular la competitividad.

Adicionalmente, se relacionan aquellos procesos que tienen un vínculo directo con la ecoinnovación. Por otro lado, la premisa del modelo la constituye: el vínculo estrecho de la proyección estratégica de la organización, el compromiso con la mejora continua sobre bases científicas y la minimización de los impactos ambientales que se asocian con el desarrollo de la actividad hotelera (Diagrama 3).

\section{Diagrama 3 \\ Modelo de Ecoinnovación para empresas de servicios de hospedaje}

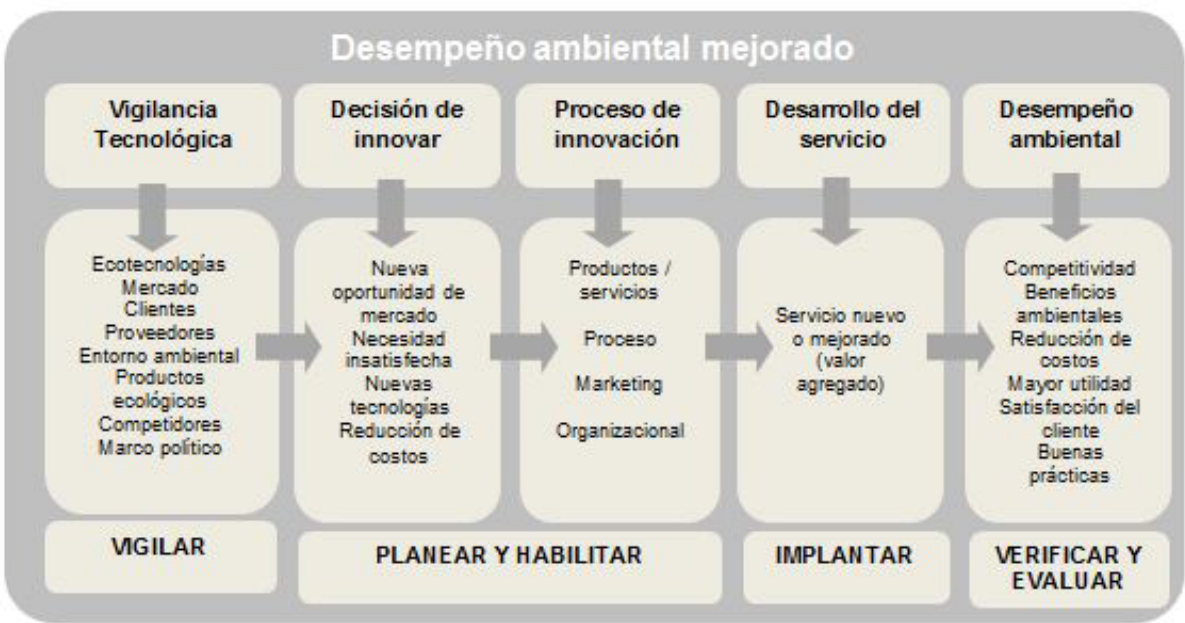

Fuente: Elaboración propia 
La función inicial de Vigilar, apoya la toma de decisiones estratégicas en materia de ecoinnovación. La vigilancia tecnológica (VT), permitirá a los establecimientos de hospedaje, actuarcon rapidez ante la detección de amenazas y aprovechar nuevas áreas de oportunidad o requerimientos de los clientes, a través de: la selección de información actualizada, búsqueda permanente de nuevas patentes y ecotecnologías, de conocimiento y análisis de la situación actual de la empresa, de información sobre nuevos avances o estudios, así como de las mejores prácticas de gestión que aplican otras empresas. El resultado final será la obtención de numerosas ideas, que pueden constituir proyectos de ecoinnovación.

Una vez efectuada la VT, se procede con la función de planear y habilitar, comenzando con el diseño de cronogramas de trabajo para la asignación de recursos. En esta etapa se determinan los proyectos de ecoinnovación a ejecutarse, a partir del análisis previo de información sobre las necesidades ambientales y tecnológicas del hotel.

Posteriormente, determinado el análisis de la problemática actual y las tendencias, así como de los proyectos de mejora ambiental factibles, se procede con la etapa de implantar. Atendiendo los cronogramas de trabajo, estrategias de mercadotecnia y ventas, se logrará la operatividad del o de los proyectos para la solución de problemas reales y de impacto económico, que mejoren la competitividad de los establecimientos de hospedaje.

Prosigue la etapa de verificar y evaluar. La evaluación sistemática de los resultados de los proyectos emprendidos, estableciendo indicadores que reflejan el impacto de los servicios y/o productos, principalmente indicadores enfocados al beneficio ambiental que consideran el carácter económico.

Finalmente, el modelo de ecoinnovación propuesto para las empresas hoteleras, se basa en la incorporación de aspectos de gestión ambiental al modelo de innovación de servicios con la finalidad de responder a los retos ambientales, como la escasez del agua, ser eficiente y racional en el empleo de recursos; esto implica disminuir el uso de recursos naturales, verificar el progreso y desempeño ambiental empresarial; para lograr la complementariedad de funciones de gestión ambiental e innovación, a través de la sistematización del proceso de innovación en dichos establecimientos.

\section{Conclusiones}

Existe escasa literatura e investigaciones que documenten la relación entre la gestión de la innovación $y$ el sector servicios, y mucho menos vinculada al sector turístico; por lo que esta investigación aporta evidencia significativa sobre los modelos de gestión de la innovación en el sector servicios.

Gran parte de la tecnología y/o innovaciones necesarias para las empresas de hospedaje, ya están disponibles, pero el acceso, vinculación al conocimiento y las habilidades necesarias dentro de las empresas no es automático y genera un costo. Por lo que, estas empresas requieren de un modelo de ecoinnovación para gestionar adecuada y eficientemente sus capacidades dinámicas, en particular las que forman la capacidad de los recursos humanos para absorber tecnologías y crear mejoras en consonancia con las circunstancias de la organización. 
De la innovación a la ecoinnovación. Gestión de servicios en empresas hoteleras Velázquez-Castro, Judith Alejandra y Vargas-Martínez, Elva Esther

Cabe resaltar, que en el sector servicios, los recursos humanos son la base de la innovación; y se refuerzan con la integración de conocimientos y con el cambio de estructuras que sustentan sus hábitos, prácticas y normas. Es una oportunidad para las empresas de servicios, aprovechar sus conocimientos y capacidad creativa para contribuir a los objetivos de la organización, de manera equitativa y eficaz, a través de la gestión de la innovación.

Los modelos de gestión de las empresas de servicio han evolucionado. Hoy en día, las empresas han pasado de los modelos de gestión tradicionales a modelos más dinámicos. Los modelos actuales consideran la eficiencia y la calidad sobre el simple hecho de la prestación del servicio. En las empresas del sector hotelero, los modelos de gestión se adaptan a las tendencias globales y necesidades de los clientes; por lo que también se han tomado algunas medidas para conseguir actividades turísticas con el menor impacto ambiental, tales como la importancia de concientizar a los prestadores de servicios sobre la preservación y uso eficiente de los recursos naturales, de los que dependen inherentemente estas empresas.

No obstante, son escasos los modelos de innovación en servicios que consideran los aspectos ambientales; por lo que la esta propuesta se basó en la incorporación de aspectos de gestión ambiental al modelo de innovación de servicios, logrando la complementariedad de sus funciones (ambientales y de innovación). Se incluyen aspectos que van desde la vigilancia tecnológica (vigilar), pasando por la decisión de innovar y el proceso que esto exige (planear y habilitar), hasta el desarrollo del servicio (implantar), y la fase de desempeño ambiental, que implica verificar y evaluar aspectos como beneficios ambientales, buenas prácticas, satisfacción del cliente, entre otros.

En lo que respecta a las empresas de hospedaje, éstas tienen que gestionar sus recursos y estar al tanto de los nuevos avances científicos y tecnológicos para mejorar sus procesos; ya que el resultado será sin duda, una empresa bien gestionada y con resultados favorables, tanto para sus accionistas como para la naturaleza.

Cabe indicar que esta propuesta para la gestión de ecoinnovación en las empresas de hospedaje, tiene pertinencia y vigencia; y que además puede ser utilizada y adaptada por otro tipo de empresas prestadoras de servicios. Asimismo, se verifica su solidez conceptual y metodológica, no obstante, puede ser mejorado y validar su utilidad práctica empresarial a partir de su aplicación, para diseñar un plan de fortalecimiento a la gestión ambiental y de innovación.

\section{Referencias bibliográficas}

Aguilar, Gabriel; Herrera, Liliana y Baca, Ernesto (2013), Capacidad de Absorción: Estructura y Funcionalidad para el Sector Servicios.

Arundel, Anthony y Kemp, René (2009), Measuring eco-innovation. Working Paper Series. United Nations University, UNU.

ATKearney (2007), Innovation Management Assessment. En http://www.atkearney.de/content/ misc/wrapper.php/name/file at kearney_innovation_management assessment 11 $\overline{9} 462882 . \mathrm{pdf}$ (Consultado el 1 de junio de 2014).

Betancourt, Agüero y Mayo, Alegre (2010), La evaluación de la cal idad de 
servicio. En: http://www.eumed.net/ ce/2010/ (Consultado el 12 de junio de 2014).

Camisón, César; Cruz, Sonia y González, Tomás (2007), Gestión de la calidad: conceptos enfoques, modelos y sistemas. Editorial Prentice Hall, Madrid.

Días, Reinaldo (2008), Marketing ecológico y turismo. Estudios y perspectivas en turismo, Vol. 17, No. 2, pp. 140-155.

EKOS Negocios (2011), Innovación. Los países más innovadores del mundo. Revista electrónica, 209. En: http:// www.ekosnegocios.com/revista/ pdf/209.pdf (Consultado el 13 de abril de 2014).

Finnish Funding Agency for Technology and Innovation, TEKES (2014), Funding for the best ideas. En: http://www. tekes.fi/en/ (Consultado el 1 de junio de 2014).

Fundación COTEC para la Innovación Tecnológica, (2010), La innovación en un sentido amplio: un modelo empresarial. Análisis conceptual y empírico. Colección: Innovación Práctica.

Hellström, Tomas (2007), Dimensions of Environmentally Sustainable Innovation. Sustainable Development, No. 15, pp. 148-159.

Hernández, Tirso y García, María (2011), La Responsabilidad Social Empresarial en el Valle del Mezquital, del Estado de Hidalgo. Primer Foro de Investigación de las Universidad Tecnológicas, en el área económico-administrativa.

Hertog, Pim y Bilderbeek, Rob (1998), Conseptualizing (service) innovation and the knowledge flow between kibs and their clients. SI4S Topical Paper.
Ministerio de Industria y Comercio de Noruega (2006), Innovation in services: typology, case studies and policy implications. ECON-Report No. 2006-025.

Organización Mundial Del Turismo, OMT (1998), Turismo panorama 2020. Nuevas previsiones. OMT. Madrid.

Hinojosa, Antonio (2006), Innovación de Procesos. Cuadernos de Gestión Tecnológica, Premio Nacional de Tecnología, PNT México.

Organización para la Cooperación y el Desarrollo Económicos, OCDE (2012) Education Indicators Focus. En: http://www.oecd.org/education/ skills-beyond-school/49729932.pdf (Consultado el 3 de junio de 2014).

Premio Nacional de Tecnología e innovación, PNT (2012), Modelo Nacional de Gestión de Tecnología e Innovación. México.

Schilling, Annika y Werr, Andreas (2009), Managing and Organizing for Innovation in Service Firms. A literature review with annotated bibliography. Vinnova Report.

Velázquez, Judith y Vargas, Elva (2014), Ecoinnovación en turismo: una aproximación al estado de la cuestión. Revista Gestión y Ambiente, Vol. 17, No. 1, pp. 191-207.

World Commision on Environment and Development, WCED (1987). Our Common Future (Brundtlland Report), United Nations.

Zahra, Shaker y George, Gerard. (2002), Absorptive capacity: A review, reconceptualization, and extension. Academy of Management. The Academy of Management Review, Vol. 27, No. 2, pp. 185-203. 